\title{
Do Fetal Monitoring Tests predict Adverse Perinatal Outcomes in Pregnant Women with Absent End Diastolic Flow? A Retrospective Study
}

\author{
${ }^{1}$ Sailaja Devi Kallur, ${ }^{2}$ Nuzhat Aziz
}

\begin{abstract}
Aim: To determine the ability of fetal monitoring tests to predict adverse perinatal outcomes in absent end diastolic flow (AEDF) babies.

Materials and methods: A retrospective cohort study of pregnant women with AEDF during the period 2001 to 2009. Fetal monitoring tests of interest included amniotic fluid index (AFI), nonstress tests (NST), and Doppler flow studies. Adverse perinatal outcomes included perinatal/neonatal mortality, necrotizing enterocolitis, respiratory distress syndrome, and grades III/IV intraventricular hemorrhage. Sensitivity, specificity, likelihood ratios, adjusted odds ratios, area under the receiver operator characteristic curves (AUROC) and the 95\% confidence intervals were determined.

Study included 142 women with AEDF who delivered before 34 weeks. Indications for delivery included abnormal AFI in $6(4.23 \%)$, worsening Doppler in $31(21.83 \%)$, and abnormal NST in $48(33.80 \%)$. An adverse fetal event was noted in 107 [75.35\%, 95\% confidence interial (Cl) $68.18 \%, 82.53 \%$ ]. Birth weight adjusted odds for an adverse perinatal outcome decreased (Odds ratio: $0.79,95 \% \mathrm{Cl}: 0.56,1.10, p=0.16$ ) with an increase in each week of gestation. Fetal monitoring tests did not have clinically meaningful positive/negative likelihood ratio or significant AUROC.
\end{abstract}

Conclusion: Current fetal monitoring tests are more useful to identify noncompromised fetuses than to identify fetal distress. Delaying delivery till 34 weeks might improve outcomes.

Keywords: Absent end diastolic flow, Fetal monitoring, Diagnostic tests, Perinatal outcomes, Neonatal death.

How to cite this article: Kallur SD, Aziz N. Do Fetal Monitoring Tests predict Adverse Perinatal Outcomes in Pregnant Women with Absent End Diastolic Flow? A Retrospective Study. Int J Infertil Fetal Med 2014;5(1):22-26.

Source of support: Nil

Conflict of interest: None

Date of Received: 31-09-2013

Date of Acceptance: 04-04-14

Date of Publication: April 2014

\section{INTRODUCTION}

Absent end diastolic flow (AEDF) in the umbilical artery during pregnancy indicates severe compromise of the

\footnotetext{
${ }^{1,2}$ Consultant Obstetrician

1,2Department of Obstetrics Medicine, Fernandez Hospital Pvt. Ltd., Hyderabad, Andhra Pradesh, India
}

Corresponding Author: Nuzhat Aziz, Consultant Obstetrician Department of Obstetrics Medicine, Fernandez Hospital Pvt Ltd Hyderabad, Andhra Pradesh, India, e-mail: aziznuzhat@gmail.com fetoplacental circulation and is an indication for immediate and continuous fetal monitoring due to a higher risk for adverse perinatal outcomes. ${ }^{1-4}$ Previous studies have suggested that increasing gestational age and consequently increasing birth weight are good predictors of intact survival, although the proportion of women with AEDF was not significantly different between survivors and nonsurvivors. ${ }^{5}$ An obstetrician, in the presence of AEDF in pregnancy, has to determine an appropriate time for delivery of the fetus based on gestational age, evidence of fetal compromise, and birth weight to optimize neonatal outcomes. The determination of the time of delivery is often based on the results of the various tests for fetal monitoring that include nonstress test (NST), amniotic fluid index (AFI), and Doppler flow studies. We did a retrospective study to determine the ability of fetal monitoring to predict adverse perinatal outcomes in pregnant women with AEDF in a singleton pregnancy and intrauterine growth restriction presenting at an advanced tertiary perinatal care center between 2001 and 2009.

\section{MATERIALS AND METHODS}

The study protocol was part of regular AEDF audit and adhered to the tenets of the Declaration of Helsinki. The study institute is an advanced tertiary care referral perinatal center located at Hyderabad, India. Each pregnant woman attending the antenatal clinic at the study institute undergoes a standardized antenatal examination that includes demographic information, past and current obstetric and medical history, personal and risk history, clinical examination including trimester specific ultrasound examination for nuchal thickness, fetal abnormalities, and fetal wellbeing. The institute has a fetal medicine unit with a group of trained and licensed obstetric sonologists specialized in fetal medicine that performs fetal monitoring on compromised fetuses. Compromised fetuses or fetuses at risk for compromise are monitored by serial NST and serial Doppler umbilical artery and fetal vessel studies. A NST was considered abnormal if it showed a lack of acceleration for a minimum period of 40 minutes with decreased beat-to-beat variability and unprovoked decelerations. Fetuses with absent blood flow in diastolic component of fetal cardiac cycle in the umbilical artery on Doppler studies were considered to 
have AEDF. Sonographic fetal biometry and Doppler were performed using transabdominal transducers $(3.5-5 \mathrm{MHz})$ and Voluson 730 expert machine. An AFI $<5$ cm was considered as abnormal and labeled oligohydramnios. The fetal surveillance protocol for compromised or growth restricted fetuses is almost similar except if there is a worsening of maternal indications for delivery (example-preeclampsia). Pregnant women detected with AEDF undergo daily NSTs, AFI, and Doppler flow studies if the gestational age at detection of AEDF is $<34$ weeks and the initial test results are reassuring or do not show worsening fetal compromise. The fetus is delivered immediately in the presence of worsening fetal compromise (nonreactive, decelerative NSTs and AFI $<5 \mathrm{~cm}$ or worsening of Doppler flow studies-reversed end diastolic flow in umbilical artery, middle cerebral artery redistribution or abnormal ductus venosus) or if the gestational age has reached 34 weeks.

We identified the records of pregnant women with a singleton pregnancy, an estimated fetal weight $<5$ th centile for the gestational age, and an AEDF on umbilical artery Doppler from an electronic database that registers maternal and neonatal information at our institute. Pregnant women with multiple pregnancies and/or fetuses with structural abnormalities were not included in this analysis. We extracted data on maternal sociodemographics, medical and obstetric history, antenatal course, results of the serial ultrasound examinations, other investigations, intrapartum course, details of delivery, and immediate neonatal outcomes. Neonatal outcomes of interest included necrotizing enterocolitis (NEC), respiratory distress syndrome (RDS), Grade 3 and 4 intraventricular hemorrhages (IVH), and neonatal mortality and were based on standard definitions applied by a neonatologist. A composite adverse perinatal outcome (APO) was defined as the presence of at least one of the four neonatal outcome of interest. We excluded all mothers who were delivered at 34 weeks gestation, since the protocol for management of AEDF demanded a delivery at completion of 34 weeks. The abnormal fetal test responsible for the decision to deliver was then evaluated for its ability to predict adverse neonatal outcomes.

We used STATA version 9.0 (College Station, Tx, USA) for the statistical analysis. We used nonparametric tests to compare the means of continuous variables (K sample equality of median test with continuity corrected Pearson chi-square) and the Mann-Whitney test to compare categorical variables as the data were not normally distributed. Normality was tested using the Shapiro-Wilk test for normality. Associations with adverse perinatal outcomes were initially explored using a bivariate model and then in a multivariate model that included factors found significant in the bivariate regression model. Odds ratios and the $95 \%$ confidence intervals around the ORS were determined. The performance of each of the fetal monitoring tests with reference to perinatal outcomes (point estimates and 95\% confidence intervals around the point estimates) was assessed by the sensitivity, specificity, positive and negative likelihood ratios, and the area under the reciever operating charateristics curve (AUROC). The likelihood ratio essentially expresses the odds that a given level of a diagnostic test result would be expected in a patient with the target disorder compared with a person without the target disorder. The 'positive likelihood ratio' (LR+) tells us how much to increase the probability of disease if the test is positive and is used to 'rule in' disease. Generally, a $\mathrm{LR}+>10$ is considered useful to rule in disease. The 'negative likelihood ratio' (LR-) tells us how much to decrease the probability of disease if the test is negative and is used to rule out disease. Generally, a LR - of $<0.1$ is considered useful to rule out disease. We preferred the LRS rather than predictive values as the LRS are more relevant for application to an individual patient at a clinic/hospital, while predictive values are more useful for a general population.

\section{RESULTS}

We identified 224 pregnant women with AEDF between 2001 and 2009 based on an ultrasound examination in the third trimester. A total of 82 (36.61\%) of these 224 women were excluded from the analysis as they were referrals who presented to our hospital beyond 34 weeks of gestation. We also excluded those who were monitored with these tests and were delivered as per protocol on reaching 34 weeks of pregnancy. The records of 142 women with AEDF were considered for analysis.

The mean age standard deriation (SD) of women in the study was 26.99 (4.65) years and 85 (59.86\%) were nulliparous (Table 1). The indications for delivery included an abnormal AFI $(<5 \mathrm{~cm})$ in $6[4.23 \%, 95 \%$ confidence interval (CI) $0.88 \%, 7.57 \%$, worsening Doppler in $31(21.83 \%, 95 \%$ CI: $14.95 \%, 28.71 \%)$, and an abnormal NST in $48(33.80 \%$, $95 \%$ CI: $25.93 \%, 41.68 \%$ ) of pregnant women with AEDF in this series. One pregnancy was terminated and the remaining 141 women underwent a cesarean section for delivery. The

Table 1: Characteristics of 224 pregnant women with absent end diastolic flow

\begin{tabular}{ll}
\hline Mean age (SD), median, & $26.99(4.65), 26.00$, \\
range in years & $19.00-40.00$ \\
\hline Nulliparous & $85(59.86 \%)$ \\
Diabetes & $10(7.09 \%)$ \\
Hypertension & $7(4.97 \%)$ \\
Preeclampsia & $98(70.50 \%)$ \\
Mean gestational age (SD), median & $30.85(1.68), 31.00$, \\
range in weeks at diagnosis & $26.00-33.00$ \\
\hline
\end{tabular}

SD: Standard deviation 
Table 2: Adverse perinatal outcomes stratified by gestational age

\begin{tabular}{lllll}
\hline Gestational age & NEC $(n=12)$ & IVH-grade 3 or 4 $(n=2)$ & $R D S(n=105)$ & $N N D(n=22)$ \\
\hline$<30$ weeks & $2(16.67 \%)$ & $1(50.50 \%)$ & $25(23.81 \%)$ & $8(36.36 \%)$ \\
30 to $<32$ weeks & $6(50.00 \%)$ & $1(50.50 \%)$ & $43(40.95 \%)$ & $10(45.45 \%)$ \\
32 to $<34$ weeks & $4(33.33 \%)$ & - & $37(35.24 \%)$ & $4(18.18 \%)$ \\
\hline
\end{tabular}

IVH: Intraventricular hemorrhage; NEC: Necrotizing enterocolitis; NND: Neonatal death; RDS: Respiratory distressed syndrome

Table 3: Maternal and fetal factors with adverse perinatal outcome

\begin{tabular}{llll}
\hline Parameter & $A P O(n=107)$ & Without $A P O(n=35)$ & Statistical test, $p$-value \\
\hline Mean (SD), median age of mother & $26.93(4.69), 26.00$ & $27.17(4.59), 26.00$ & K-sample equality of median test, $\mathrm{p}=0.63$ \\
Diabetes & $9(8.41 \%)$ & $1(2.94 \%)$ & Mann-Whitney test, $\mathrm{p}=0.28$ \\
Hypertension & $7(6.54 \%)$ & $0(0.0 \%)$ & Mann-Whitney test, $\mathrm{p}=0.12$ \\
Preeclampsia & $73(69.52 \%)$ & $25(73.53 \%)$ & Mann-Whitney test, $\mathrm{p}=0.66$ \\
Steroids & $105(98.13 \%)$ & $34(97.14 \%)$ & Mann-Whitney test, $\mathrm{p}=0.73$ \\
Surfactant & $14(13.08 \%)$ & $1(2.86 \%)$ & Mann-Whitney test, $\mathrm{p}=0.09$ \\
Mean (SD), median gestational & $30.60(1.61), 30.00$ & $31.63(1.66), 32$ & K-sample equality of median test, $\mathrm{p}=0.001$ \\
age at delivery (weeks) & & & \\
Mean (SD), median birth weight & $1.07(0.24), 1.08$ & $1.24(0.28), 1.30$ & K-sample equality of median test, $\mathrm{p}=0.03$ \\
\hline
\end{tabular}

APO: Apolipoprotein B, SD: Standard deviation

mean (SD) diagnosis-to-delivery interval was 2.09 (3.12) days (median 1.00 day).

One fetus was still born and 22 babies died during the neonatal period for a perinatal mortality rate of 162 per 1000 live births. Nine babies were lost to follow-up including seven who took a discharge against medical advice and were not considered for the determination of the perinatal mortality rate. The mean (SD) birth weight was $1.11(0.26) \mathrm{kg}$ (median $1.10 \mathrm{~kg}$ ). A total of $105(75.00 \%$, 95\% CI: $67.74 \%, 82.26 \%)$ babies had RDS, $11(7.80 \%$, 95\% CI: $3.32 \%, 12.28 \%$ ) babies had a low APGAR score at 5 minutes, 12 (8.51\%, 95\% CI: 3.85\%, 13.17\%) babies had NEC, and two $(1.42 \%, 95 \%$ CI: $0.56 \%, 3.39 \%)$ babies had grade 3 or 4 IVH (Table 2). An adverse fetal event was noted in $107(75.35 \%, 95 \%$ CI: $68.18 \%, 82.53 \%)$ of the 142 pregnancies with AEDF.

Birth weight was significantly lower in babies with adverse perinatal outcomes (K sample equality of median test $p=0.03$ ) compared with babies without adverse perinatal outcomes (Table 3 ). Babies with adverse perinatal outcomes had a lower (K sample equality of median test $p=0.001$ ) mean gestational age at delivery (see Table 3 ). The adjusted odds for an adverse perinatal outcome decreased with an increase in each week of gestation (birth weight adjusted OR: $0.79,95 \%$ CI: $0.56,1.10$ ) although this was not statistically significant $(\mathrm{p}=0.16)$.

The sensitivity of the various fetal monitoring tests was low although AFI and Doppler had good specificity in determining adverse fetal outcomes. None of the fetal monitoring tests had a clinically meaningful positive or negative likelihood ratio or a clinically significant area under ROC curves (Table 4). None of the fetal monitoring tests were predictive of specific adverse fetal outcomes (Table 5).
After adjusting for birth weight and gestational age, NEC was not associated with oligohydramnios (adjusted OR: 3.98, 95\% CI: 0.58, 27.55, $\mathrm{p}=0.16$ ), worsening Doppler (adjusted OR: $0.92,95 \%$ CI: $0.22,3.89, p=0.91$ ), or abnormal NST (adjusted OR: 0.73, 95\% CI: 0.18, 2.95). After adjusting for gestational age, RDS was not associated with worsening Doppler flow studies (adjusted OR: 0.64, 95\% CI: 0.24, 1.69, $\mathrm{p}=0.37$ ) or abnormal NST (adjusted OR: $0.56,95 \%$ CI: 0.25 , $1.27, \mathrm{p}=0.16$ ). After adjusting for gestational age, neonatal death was not associated with worsening Doppler (adjusted OR: $1.03,95 \%$ CI: $0.32,3.32, p=0.96$ ) or abnormal NST (adjusted OR: 1.17, 95\% CI: 0.38, 3.55, $\mathrm{p}=0.79$ ).

\section{DISCUSSION}

The results of this study indicate that current antepartum fetal monitoring tests are not particularly useful to discriminate or predict fetuses at risk for an adverse outcome in a high-risk group of pregnant women with AEDF in umbilical artery. We could not find evidence for an optimal antepartum test predicting adverse perinatal outcomes that will help to determine delivery for the growth restricted fetus with AEDF. ${ }^{6}$ The sensitivity, likelihood ratios, and AUROC of all the tests were not within clinically acceptable limits although AFI $<5 \mathrm{cms}$ showed a very good specificity for any adverse perinatal outcome. Fetal monitoring tests appear to be more useful in identifying noncompromised fetuses rather than fetuses at risk for adverse events.

The timing of delivery of the fetus for an optimal outcome in women with AEDF remains unclear in the absence of a fetal monitoring test that can predict adverse outcomes. We found that the adjusted odds for an adverse perinatal outcome decreased for each week increase in gestational age although 
Table 4: Diagnostic or predictive ability of fetal monitoring tests to predict abnormal fetal outcomes in 142 pregnant women with absent end diastolic flow

\begin{tabular}{llllll}
\hline Test & Sensitivity (\%) & Specificity (\%) & Positive likelihood ratio & Negative likelihood ratio & $\begin{array}{l}\text { Area under } \\
\text { ROC }\end{array}$ \\
\hline Amniotic fluid index & 5.61 & & & & 0.53 \\
$<5$ & $(2.09,11.80)$ & $(90.00$ & 4.33 & $(0.90,1.01)$ & $(0.51,0.55)$ \\
Worsening Doppler & 19.60 & 71.40 & 0.69 & 1.13 & 0.46 \\
& $(12.60,28.40)$ & $(53.70,85.40)$ & $(0.36,1.31)$ & $(0.89,1.42)$ & $(0.37,0.54)$ \\
Abnormal nonstress & 29.90 & 54.30 & 0.65 & 1.29 & 0.42 \\
test & $(21.40,39.50)$ & $(36.60,71.20)$ & $(0.41,1.04)$ & $(0.93,1.79)$ & $(0.33,0.52)$ \\
\hline
\end{tabular}

Data in parenthesis are the $95 \%$ confidence intervals. ROC: Receiver operating characteristics

Table 5: Diagnostic or predictive ability of fetal monitoring tests to predict specific abnormal fetal outcomes in pregnant women with absent end diastolic flow

\begin{tabular}{llll}
\hline Test & Outcome & Likelihood ratio + & Area under ROC \\
\hline Amniotic fluid index $<5 \mathrm{~cm}$ & NEC & $5.38(1.1,26.4)$ & $0.57(0.46,0.68)$ \\
Worsening Doppler & NEC & $1.15(0.41,3.24)$ & $0.52(0.38,0.65)$ \\
Abnormal nonstress test & NEC & $0.72(0.26,1.96)$ & $0.45(0.32,0.58)$ \\
Amniotic fluid index $<5$ & IVH Grade 3 or 4 & $3.59(0.26,50.3)$ & $0.48(0.46,0.50)$ \\
Worsening Doppler & IVH Grade 3 or 4 & $2.32(0.56,9.6)$ & $0.64(0.15,1)$ \\
Abnormal nonstress test & IVH Grade 3 or 4 & $1.48(0.36,6.03)$ & $0.58(0.09,1.00)$ \\
Amniotic fluid index $<5$ & Respiratory distress & $4.42(0.25,76.40)$ & $0.53(0.51,0.55)$ \\
Worsening Doppler & Respiratory distress & $0.78(0.39,1.54)$ & $0.47(0.39,0.55)$ \\
Abnormal nonstress test & Respiratory distress & $0.67(0.42,1.06)$ & $0.42(0.33,0.52)$ \\
Amniotic fluid index $<5$ & Neonatal death & $0.38(0.02,6.42)$ & $0.47(0.45,0.49)$ \\
Worsening Doppler & Neonatal death & $1.35(0.64,2.83)$ & $0.53(0.43,0.63)$ \\
Abnormal nonstress test & Neonatal death & $0.84(0.42,1.70)$ & $0.47(0.37,0.57)$ \\
\hline
\end{tabular}

Data in parenthesis are the $95 \%$ confidence intervals; NEC: Necrotizing enterocolitis; IVH: Intraventricular hemorrhage; ROC: Receiver operating characteristics

this decrease was not statistically significant $(p=0.16)$. We did find that birth weight was significantly lower for fetuses with adverse perinatal outcomes. These results might suggest that delaying delivery to improve birth weight (assuming an increase in birth weight with increasing gestational age) may be a useful option in the management of pregnant women with AEDF. ${ }^{7}$ However, in the absence of a test that can predict adverse fetal outcomes, ${ }^{8}$ an approach that focuses on delaying delivery is fraught with danger especially when one considers that three-fourths of pregnant women with AEDF in this study had an adverse perinatal fetal event. It might be a good idea to use AFI $>5 \mathrm{~cm}$ to identify noncompromised fetuses considering that the results of this study indicate that an apparently normal AFI has a very good specificity to identify noncompromised fetuses. Given the high specificity of AFI $<5 \mathrm{~cm}$, oligohydramnios is to be considered as a possible indication of fetal compromise although the sensitivity, likelihood ratios, and area under the curve of AFI do not suggest a good ability to predict adverse outcomes.

An additional consideration is the association of AEDF or abnormal Doppler with placental injury rather than fetal injury. ${ }^{9}$ Increased resistance to blood flow within the placenta has consequences on the supply of nutrients to the fetus with short-term and long-term consequences on fetal health. With improving standards of neonatal care, it is probable that obstetricians might prefer earlier delivery of a live preterm baby rather than lose the fetus in utero especially in the absence of reasonably accurate tests to predict adverse outcomes.

We used the $<5$ th centile of gestational age specific estimated fetal weight to determine severe fetal growth restriction in this study. However, we do not expect this cut-off to influence the results as most adverse perinatal events are in a population with an estimated fetal weight $<5$ th centile compared with a population based on a $<10$ th centile gestational age-specific estimated fetal weight. Previous studies have reported that low estimated fetal weights, abnormal Doppler and oligohydramnios are predictive of poor neonatal outcomes. ${ }^{10,11} \mathrm{We}$ found similar results with a high incidence of adverse perinatal outcomes in women with AEDF.

The retrospective design of this study is a limitation as we could not control for all possible confounders. Adverse neonatal outcomes may be attributable to a combination of hypoxia, prematurity, and other factors. However, the retrospective nature of data was a limitation in attributing 
a specific primary cause for the adverse neonatal event. The strength of the study is the documentation and the availability of regularly updated standardized protocols for antenatal and intrapartum care. Prospective studies, especially multicentric randomized controlled trials, are necessary to determine further evidence for optimal fetal monitoring tests in pregnant women with AEDF and to determine an optimal strategy to deliver the fetus including benefit-risk ratios of delaying delivery even by a few gestational weeks.

\section{CONCLUSION}

Current fetal monitoring tests are more useful to identify non compromised fetuses than to conclusively provide evidence of fetal distress in pregnant women with AEDF. Delaying the delivery, in the absence of maternal or fetal indications of distress, till 34 weeks of gestation might improve outcomes in babies.

\section{REFERENCES}

1. Resnik R. Intrauterine growth restriction. Obstet Gynecol 2002;99(3):490-496.

2. Zelop CM, Richardson DK, Heffner LJ. Outcomes of severely abnormal umbilical artery Doppler velocimetry in structurally normal singleton fetuses. Obstet Gynecol 1996;87(3): 434-438.

3. Karsdrop VH, van Vugt JM, van Geijn HP, Kostense PJ, Ardyini D, Montenegro N, Todros T. Clinical significance of absent or reversed end diastolic velocity waveforms in umbilical artery. Lancet 1994;344(8938):1664-1668.

4. Kingdom JC, Burrell SJ, Kaufmann P. Pathology and clinical implications of abnormal umbilical artery Doppler waveforms. Ultrasound Obstet Gynecol 1997;9(4):271-286.

5. Baschat AA, Cosmi E, Bilardo CM, Wolf H, Berg C, Rigano S, Germer U, Moyano D, Turan S, Hartung J, et al. Predictors of neonatal outcome in early-onset placental dysfunction. Obstet Gynecol 2007 Feb;109(2 Pt 1):253-261.

6. Odibo AO, Quinones JN, Lawrence-Cleary K, Stamilio DM, Georges GA. What antepartum fetal test should guide the timing of delivery of the preterm growth-restricted fetus? A decision analysis. Am J Obstet Gynecol 2004;191(4):1477-1482.

7. Mclntire DD, Bloom SL, Casey BM, Leveno KJ. Birth weight in relation to morbidity and mortality among newborn infants. N Engl J Med 1999;340(16):1234-1238.

8. Gonzalez JM, Stamilio DM, Ural S, Macones GA, Odibo AO. Relationship between abnormal fetal testing and adverse perinatal outcomes in intrauterine growth restriction. Am J Obstet Gynecol 2007;196(5):e-48-e51.

9. Dicke JM, Huettner P, Yan S, Odibo A, Kraus FT. Umbilical artery Doppler indices in small for gestational age fetuses: correlation with adverse outcomes and placental abnormalities. J Ultrasound Med 2009 Dec;28(12):1603-1610.

10. Craigo SD, Beach ML, Harvey-Wilkes KB, D'Alton ME. Ultrasound predictors of neonatal outcome in intrauterine growth restriction. Am J Perinatol 1996 Nov;13(8):465-471.

11. Soregaroli M, Bonera R, Danti L, Taddei F, Valcamonico A, Frusca T. Prognostic role of umbilical artery Doppler velocimetry in growth restricted fetuses. J Matern Fetal Neonatal Med 2002 Mar;11(3):199-203. 